\title{
Multiple periodic solutions for delay differential equations with a general piecewise constant argument
}

\author{
Hui-Sheng Ding ${ }^{a, *}$, Hong Wang ${ }^{a}$, Gaston M. N'Guérékata ${ }^{b}$ \\ ${ }^{a}$ College of Mathematics and Information Science, Jiangxi Normal University, Nanchang, Jiangxi 330022, P. R. China. \\ ${ }^{b}$ Department of Mathematics, Morgan State University, 1700 E. Cold Spring Lane, Baltimore, M.D. 21251, USA.
}

Communicated by $\mathrm{X}$. Liu

\begin{abstract}
This paper is concerned with the existence of multiple periodic solutions for some delay differential equations with a general piecewise constant argument. Under some sufficient conditions, we establish the existence of two and three nonnegative periodic solutions for the addressed delay differential equation with piecewise constant argument. Also, we apply one of our main results to a Nicholson's blowflies type model. (C)2017 All rights reserved.
\end{abstract}

Keywords: Piecewise constant, periodic solution, multiple periodic solution. 2010 MSC: 34C25.

\section{Introduction and preliminaries}

Throughout this paper, we denote by $\mathbb{N}$ the set of all positive integers, by $\mathbb{Z}$ the set of all integers, by $\mathbb{R}$ the set of all real numbers, and by $C_{T}(\mathbb{R})$ the set of all continuous $T$-periodic functions from $\mathbb{R}$ to $\mathbb{R}$, where $T>0$ is a fixed constant. Moreover, assume that the two real sequences $\left\{t_{k}\right\}_{k \in \mathbb{Z}}$ and $\left\{\gamma_{k}\right\}_{k \in \mathbb{Z}}$ satisfy the following statements:

(a) For every $k \in \mathbb{Z}, t_{k}<t_{k+1}, \lim _{k \rightarrow-\infty} t_{k}=-\infty, \lim _{k \rightarrow+\infty} t_{k}=+\infty$, and

$$
0<\inf _{k \in \mathbb{Z}}\left|t_{k+1}-t_{k}\right| \leqslant \sup _{k \in \mathbb{Z}}\left|t_{k+1}-t_{k}\right|<+\infty ;
$$

(b) for every $k \in \mathbb{Z}, \gamma_{k} \in\left[t_{k}, t_{k+1}\right]$, and there exists $l \in \mathbb{N}$ such that

$$
t_{k+l}=t_{k}+T, \quad \gamma_{k+l}=\gamma_{k}+T, \quad k \in \mathbb{Z} .
$$

The aim of this paper is to investigate the existence of multiple positive periodic solutions to the following delay differential equation with a general piecewise constant argument:

$$
x^{\prime}(t)=-a(t) x(t)+b(t) f(t, x(t-\tau(t)))+c(t) g(t, x(\gamma(t))), \quad t \in \mathbb{R},
$$

where $a, b, c \in C_{T}(\mathbb{R})$ are nonnegative functions, $f, g: \mathbb{R} \times[0,+\infty) \rightarrow[0,+\infty)$ are continuous and T-

\footnotetext{
*Corresponding author

Email addresses: dinghs@mail .ustc.edu.cn (Hui-Sheng Ding), 1394007574@qq.com (Hong Wang), Gaston. N'Guerekata@morgan.edu (Gaston M. N'Guérékata)

doi:10.22436/jnsa.010.04.54
} 
periodic about the first argument, and

$$
\gamma(t)=\gamma_{k}, \quad t \in\left[t_{k}, t_{k+1}\right), \quad k \in \mathbb{Z} .
$$

It is easy to see that if $t_{k}=k$ and $\gamma(t)=[t]$, then (1.1) reduces to a classical differential equation with piecewise constant argument.

There is a large literature on multiple positive periodic solutions for functional differential equations. However, to the best of our knowledge, it seems that there is seldom results concerning multiple periodic solutions for differential equations with piecewise constant arguments (cf. [17]).

On the other hand, differential equations with piecewise constant argument has been of great interest for many mathematicians since they have wide applications in many areas such as biomedicine, chemistry, mechanical engineering, physics, etc. Recently, several authors studied differential equations with more general piecewise constant arguments (in short DEPCAG). For the backgrounds of DEPCAG and some recent contributions on DEPCAG, we refer the reader to $[1,2,4-6,10,14-16]$ and references therein, where some interesting results on several types of DEPCAG such as the existence of solutions, the stability of solutions, the existence of periodic solutions, and the existence of almost periodic solutions, etc., are established.

Especially, in [6], Chiu et al. investigated the existence and global convergence of periodic solutions for the following differential system with piecewise constant arguments of generalized type:

$$
x_{i}^{\prime}(t)=-a_{i}(t) x_{i}(t)+\sum_{j=1}^{n}\left\{b_{i j}(t) f_{j}\left(x_{j}(t)\right)+c_{i j}(t) g_{j}\left(x_{j}(\gamma(t))\right)\right\}+I_{i}(t), \quad i=1,2, \ldots n .
$$

Stimulated by this work, in this paper, we aim to make further study on this topic, i.e., we discuss the existence of multiple positive periodic solutions. For convenience, we only consider the 1-dimensional system (1.1). We note that by using a similar proof of this paper, one can also consider some n-dimensional system such as (1.2).

Next, let us recall some definitions, notations and preliminaries. For more details, we refer the reader to $[3,11]$.

Let $X$ be a real Banach space. A closed convex set $P$ in $X$ is called a cone, if the following conditions are satisfied:

(i) if $x \in P$, then $\lambda x \in P$ for any $\lambda \geqslant 0$;

(ii) if $x \in P$ and $-x \in P$, then $x=0$.

A non-negative continuous functional $\psi$ is said to be concave on $\mathrm{P}$, if $\psi$ is continuous and

$$
\psi(\mu x+(1-\mu) y) \geqslant \mu \psi(x)+(1-\mu) \psi(y), \quad x, y \in P, \quad \mu \in[0,1] .
$$

Letting $c_{1}, c_{2}, c_{3}$ be three positive constants, and $\phi$ be a non-negative continuous functional on $P$, we denote

$$
\begin{gathered}
\mathrm{P}_{\mathrm{c}_{1}}=\left\{\mathrm{y} \in \mathrm{P}:\|y\|<\mathrm{c}_{1}\right\}, \\
\overline{\mathrm{P}_{\mathrm{c}_{1}}}=\left\{\mathrm{y} \in \mathrm{P}:\|y\| \leqslant \mathrm{c}_{1}\right\}, \\
\mathrm{P}\left(\phi, \mathrm{c}_{1}\right):=\left\{x \in \mathrm{P}: \phi(x)<\mathrm{c}_{1}\right\}, \\
\overline{\mathrm{P}\left(\phi, \mathrm{c}_{1}\right)}:=\left\{x \in \mathrm{P}: \phi(x) \leqslant \mathrm{c}_{1}\right\}, \\
\partial \mathrm{P}\left(\phi, \mathrm{c}_{1}\right):=\left\{x \in \mathrm{P}: \phi(x)=\mathrm{c}_{1}\right\}, \\
\mathrm{P}\left(\phi, \mathrm{c}_{2}, \mathrm{c}_{3}\right)=\left\{\mathrm{y} \in \mathrm{P}: \mathrm{c}_{2} \leqslant \phi(y),\|y\| \leqslant \mathrm{c}_{3}\right\} .
\end{gathered}
$$

In addition, we call that $\phi$ is increasing on $P$, if $\phi(x) \geqslant \phi(y)$ for all $x, y \in P$ with $x-y \in P$. 
Lemma 1.1 ([3]). Let $\mathrm{P}$ be a cone in a real Banach space $\mathrm{X}, \alpha$ and $\gamma$ be two increasing, nonnegative, and continuous functionals on $\mathrm{P}$, and $\theta$ be a nonnegative continuous functional on $\mathrm{P}$ with $\theta(0)=0$ such that for some $\mathrm{c}>0$ and $M>0$,

$$
\gamma(x) \leqslant \theta(x) \leqslant \alpha(x), \quad\|x\| \leqslant M \gamma(x), \quad x \in \overline{\mathrm{P}(\gamma, c)} .
$$

Moreover, suppose that there exists a completely continuous operator $\mathrm{A}: \overline{\mathrm{P}(\gamma, \mathrm{c})} \rightarrow \mathrm{P}$ and $0<\mathrm{a}<\mathrm{b}<\mathrm{c}$ such that

$$
\theta(\lambda x) \leqslant \lambda \theta(x), \quad 0 \leqslant \lambda \leqslant 1, \quad x \in \partial P(\theta, b),
$$

and

(i) $\gamma(\mathrm{Ax})>\mathrm{c}$, for all $\mathrm{x} \in \partial \mathrm{P}(\gamma, \mathrm{c})$;

(ii) $\theta(\mathrm{Ax})<\mathrm{b}$, for all $\mathrm{x} \in \partial \mathrm{P}(\theta, \mathrm{b})$;

(iii) $\mathrm{P}(\alpha, \mathrm{a}) \neq \emptyset$, and $\alpha(A x)>a$, for all $x \in \partial \mathrm{P}(\alpha, \mathrm{a})$.

Then $\mathrm{A}$ has at least two fixed points $\mathrm{x}_{1}, \mathrm{x}_{2}$ belonging to $\overline{\mathrm{P}(\gamma, \mathrm{c})}$ such that

$$
a<\alpha\left(x_{1}\right), \theta\left(x_{1}\right)<b, \text { and } b<\theta\left(x_{2}\right), \gamma\left(x_{2}\right)<c .
$$

Lemma 1.2 ([11]). Let $\mathrm{P}$ be a cone in a real Banach space $\mathrm{X}, \mathrm{c}_{4}$ be a positive constant, $\mathrm{A}: \overline{\mathrm{P}_{\mathrm{c}_{4}}} \rightarrow \overline{\mathrm{P}_{\mathrm{c}_{4}}}$ be a completely continuous mapping, and $\psi$ be a concave nonnegative continuous functional on $\mathrm{P}$ with $\psi(\mathrm{u}) \leqslant\|\mathrm{u}\|$ for all $\mathrm{u} \in \overline{\mathrm{P}_{\mathrm{c}_{4}}}$. Suppose that there exist three constants $\mathrm{c}_{1}, \mathrm{c}_{2}, \mathrm{c}_{3}$ with $0<\mathrm{c}_{1}<\mathrm{c}_{2}<\mathrm{c}_{3} \leqslant \mathrm{c}_{4}$ such that

(i) $\left\{u \in P\left(\psi, c_{2}, c_{3}\right): \psi(u)>c_{2}\right\} \neq \varnothing$, and $\psi(A u)>c_{2}$ for all $u \in P\left(\psi, c_{2}, c_{3}\right)$;

(ii) $\|\mathrm{Au}\|<\mathrm{c}_{1}$ for all $\mathrm{u} \in \overline{\mathrm{P}_{\mathrm{c}_{1}}}$;

(iii) $\psi(A u)>c_{2}$ for all $u \in P\left(\psi, c_{2}, c_{4}\right)$ with $\|A u\|>c_{3}$.

Then $\mathrm{A}$ has at least three fixed points $\mathrm{u}_{1}, \mathfrak{u}_{2}, \mathrm{u}_{3}$ in $\overline{\mathrm{P}_{\mathbf{c}_{4}}}$. Furthermore, $\left\|\mathfrak{u}_{1}\right\|<\mathrm{c}_{1}<\left\|\mathrm{u}_{2}\right\|$, and $\psi\left(\mathrm{u}_{2}\right)<\mathrm{c}_{2}<\psi\left(\mathrm{u}_{3}\right)$.

\section{Main results}

\subsection{Equivalent integral equation}

First, let us recall the following definition:

Definition 2.1. A function $x: \mathbb{R} \rightarrow \mathbb{R}$ is called a solution of (1.1) provided that

(i) $x$ is continuous on $\mathbb{R}$;

(ii) the derivative $x^{\prime}(t)$ exists at every point $t$ with the possible exception of the points $t_{k}, k \in \mathbb{Z}$, where the one-side derivatives exist;

(iii) equation (1.1) is satisfied for $x(t)$ on all intervals $\left(t_{k}, t_{k+1}\right), k \in \mathbb{Z}$, and it holds for the right derivative at the points $t_{k}, k \in \mathbb{Z}$.

Next, we prove that equation (1.1) is equivalent to an integral equation.

Lemma 2.2. Let $x \in C_{T}(\mathbb{R})$. Then $x(t)$ is a solution of equation (1.1), if and only if

$$
x(t)=\int_{t}^{t+T} G(t, s)[b(s) f(s, x(s-\tau(s))+c(s) g(s, x(\gamma(s)))] d s, \quad t \in \mathbb{R},
$$

where $\mathrm{G}(\mathrm{t}, \mathrm{s})=\frac{\mathrm{e}^{\int_{\mathrm{t}}^{\mathrm{S}} \mathrm{a}(\xi) \mathrm{d} \dot{\xi}}}{\mathrm{e}^{\int_{0}^{\top} \mathrm{a}(\xi) \mathrm{d} \xi}-1}$ for $\mathrm{t}, \mathrm{s} \in \mathbb{R}$. 
Proof. For convenience, we denote

$$
\phi(t)=e^{-\int_{0}^{t} a(s) d s}, \quad M(t)=b(t) f(t, x(t-\tau(t)))+c(t) g(t, x(\gamma(t))), \quad t \in \mathbb{R} .
$$

It is easy to see that

$$
\mathrm{G}(\mathrm{t}, \mathrm{s})=\frac{\phi(\mathrm{t}) \phi^{-1}(\mathrm{~s})}{\phi^{-1}(\mathrm{~T})-1}, \quad \frac{\partial \mathrm{G}(\mathrm{t}, \mathrm{s})}{\partial \mathrm{t}}=-\mathrm{a}(\mathrm{t}) \mathrm{G}(\mathrm{t}, \mathrm{s}), \quad \mathrm{t}, \mathrm{s} \in \mathbb{R}
$$

In addition, it follows from the assumption (b) on $\left\{t_{k}\right\}_{k \in \mathbb{Z}}$ and $\left\{\gamma_{k}\right\}_{k \in \mathbb{Z}}$ that $\gamma(t+T)=\gamma(t)$ for all $t \in \mathbb{R}$. Then, since $b, c \in C_{T}(\mathbb{R})$ and $f, g$ are T-periodic about the first argument, we conclude that $M$ is also T-periodic.

Next, we give the proof of necessary part by utilizing an idea in [9]. Let $x(t)$ be a solution of (1.1), i.e.,

$$
x^{\prime}(t)=-a(t) x(t)+M(t), \quad t \in \bigcup_{k \in \mathbb{Z}}\left(t_{k}, t_{k+1}\right),
$$

which means

$$
\frac{d}{d t}\left[\phi^{-1}(t) x(t)\right]=\phi^{-1}(t) M(t), \quad t \in \bigcup_{k \in \mathbb{Z}}\left(t_{k}, t_{k+1}\right)
$$

Noting that $x(t)$ is continuous on $\mathbb{R}$, we claim that for every $t \in \mathbb{R}$,

$$
\phi^{-1}(t) x(t)=\phi^{-1}(0) x(0)+\int_{0}^{t} \phi^{-1}(s) M(s) d s,
$$

i.e.,

$$
x(t)=\phi(t) x(0)+\phi(t) \int_{0}^{t} \phi^{-1}(s) M(s) d s .
$$

In fact, there is $k \in \mathbb{Z}$ such that $0 \in\left[t_{k-1}, t_{k}\right)$. Then, for every $t \in\left[t_{k-1}, t_{k}\right),(2.2)$ obviously holds. Also, we have

$$
x\left(t_{k}\right)=\phi\left(t_{k}\right) x(0)+\phi\left(t_{k}\right) \int_{0}^{t_{k}} \phi^{-1}(s) M(s) d s .
$$

On the other hand, for every $t \in\left[t_{k}, t_{k+1}\right)$, there holds

$$
x(t)=\phi(t) \phi^{-1}\left(t_{k}\right) x\left(t_{k}\right)+\phi(t) \int_{t_{k}}^{t} \phi^{-1}(s) M(s) d s,
$$

which together with (2.3) yields that (2.2) also holds for every $t \in\left[t_{k}, t_{k+1}\right)$. By similar arguments, one can show that (2.2) holds for every $t \in \mathbb{R}$.

Since $x(0)=x(T)=\phi(T) x(0)+\phi(T) \int_{0}^{T} \phi^{-1}(s) M(s) d s$, we get

$$
x(0)=[1-\phi(T)]^{-1} \phi(T) \int_{0}^{T} \phi^{-1}(s) M(s) d s .
$$

A substitution of (2.4) into (2.2) yields

$$
\begin{aligned}
x(t) & =\phi(t)[1-\phi(T)]^{-1} \phi(T) \int_{0}^{T} \phi^{-1}(s) M(s) d s+\phi(t) \int_{0}^{t} \phi^{-1}(s) M(s) d s \\
& =\left[\phi^{-1}(T)-1\right]^{-1} \phi(t) \int_{0}^{T} \phi^{-1}(s) M(s) d s+\phi(t) \int_{0}^{t} \phi^{-1}(s) M(s) d s \\
& =\left[\phi^{-1}(T)-1\right]^{-1} \phi(t)\left[\int_{0}^{T} \phi^{-1}(s) M(s) d s+\phi^{-1}(T) \int_{0}^{t} \phi^{-1}(s) M(s) d s-\int_{0}^{t} \phi^{-1}(s) M(s) d s\right]
\end{aligned}
$$




$$
=\left[\phi^{-1}(T)-1\right]^{-1} \phi(t)\left[\int_{t}^{T} \phi^{-1}(s) M(s) d s+\phi^{-1}(T) \int_{0}^{t} \phi^{-1}(s) M(s) d s\right] .
$$

By letting $u=s+T$, the above expression implies

$$
\begin{aligned}
x(t) & =\left[\phi^{-1}(T)-1\right]^{-1} \phi(t)\left[\int_{t}^{T} \phi^{-1}(s) M(s) d s+\phi^{-1}(T) \int_{T}^{T+t} \phi^{-1}(u-T) M(u-T) d u\right] \\
& =\left[\phi^{-1}(T)-1\right]^{-1} \phi(t)\left[\int_{t}^{T} \phi^{-1}(s) M(s) d s+\int_{T}^{T+t} \phi^{-1}(u) M(u-T) d u\right] \\
& =\left[\phi^{-1}(T)-1\right]^{-1} \phi(t) \int_{t}^{t+T} \phi^{-1}(s) M(s) d s \\
& =\int_{t}^{t+T} G(t, s) M(s) d s .
\end{aligned}
$$

Finally, we give the proof of sufficient part. Let $x(t)$ satisfy (2.1). Then, we have

$$
\begin{aligned}
\frac{x(t+\Delta t)-x(t)}{\Delta t}= & \frac{1}{\Delta t}\left[\int_{t+\Delta t}^{t+\Delta t+T} G(t+\Delta t, s) M(s) d s-\int_{t}^{t+T} G(t, s) M(s) d s\right] \\
= & \frac{1}{\Delta t} \int_{t}^{t+T}[G(t+\Delta t, s)-G(t, s)] M(s) d s+\frac{1}{\Delta t} \int_{t+T}^{t+\Delta t+T} G(t+\Delta t, s) M(s) d s \\
& -\frac{1}{\Delta t} \int_{t}^{t+\Delta t} G(t+\Delta t, s) M(s) d s,
\end{aligned}
$$

combining this with the fact that $M$ is continuous on all $\left[t_{k}, t_{k+1}\right), k \in \mathbb{N}$, by using the Lebesgue dominated convergence theorem, for every $t \in \bigcup_{k \in \mathbb{Z}}\left(t_{k}, t_{k+1}\right)$, we have

$$
\begin{aligned}
x^{\prime}(t) & =\int_{t}^{t+T} \frac{\partial G(t, s)}{\partial t} M(s) d s+G(t, t+T) M(t)-G(t, t) M(t) \\
& =-a(t) x(t)+M(t) .
\end{aligned}
$$

Similarly, one can also show that

$$
x_{+}^{\prime}\left(t_{k}\right)=-a\left(t_{k}\right) x\left(t_{k}\right)+M\left(t_{k}\right), \quad k=1,2, \cdots .
$$

This means that $x(t)$ is a solution of (1.1).

\subsection{Existence of two positive solutions}

In this section, we apply Lemma 1.1 to establish an existence result about two positive periodic solutions of (1.1). Let

$$
M_{1}=\frac{1}{e^{\int_{0}^{T} a(\xi) d \xi}-1}, \quad M_{2}=\frac{e^{\int_{0}^{T} a(\xi) d \xi}}{e^{\int_{0}^{T} a(\xi) d \xi}-1} .
$$

For convenience, we list some assumptions:

(H1) There exists a constant $c_{1}>0$ such that

$$
\int_{0}^{T}\left[b(s) \inf _{c_{1} \leqslant u \leqslant \frac{c_{1}}{\delta}} f(s, u)+c(s) \inf _{c_{1} \leqslant u \leqslant \frac{c_{1}}{\delta}} g(s, u)\right] d s>\frac{c_{1}}{M_{1}} .
$$

(H2) There exists a constant $b_{1}>0$ such that

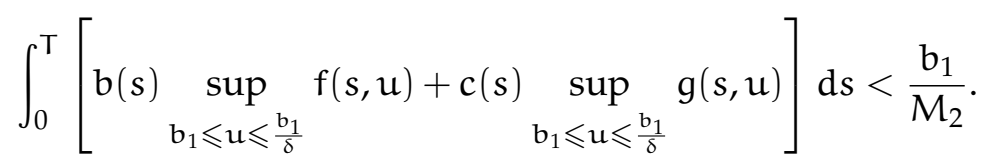


(H3) There exists a constant $a_{1}>0$ such that

$$
\int_{0}^{T}\left[b(s) \inf _{0 \leqslant u \leqslant a_{1}} f(s, u)+c(s) \inf _{0 \leqslant u \leqslant a_{1}} g(s, u)\right] d s>\frac{a_{1}}{M_{1}} .
$$

Theorem 2.3. Assume that there exist three constants $\mathrm{a}_{1}, \mathrm{~b}_{1}, \mathrm{c}_{1}$ with $0<\mathrm{a}_{1}<\mathrm{b}_{1}<\mathrm{c}_{1}$ such that (H1)-(H3) hold. Then (1.1) has at least two nonnegative T-periodic solutions.

Proof. It is easy to see that

$$
M_{1}=G(t, t) \leqslant G(t, s) \leqslant G(t, t+T)=M_{2}, \quad t \in \mathbb{R}, \quad t \leqslant s \leqslant t+T .
$$

Denote $\delta=\frac{M_{1}}{M_{2}}$ and

$$
P=\left\{y \in C_{T}(\mathbb{R}): y(t) \geqslant \delta\|y\|, t \in \mathbb{R}\right\},
$$

where $\|y\|=\sup _{t \in[0, T]}|y(t)|$. It is not difficult to verify that $P$ is a cone in $C_{T}(\mathbb{R})$.

Next, we will verify that all the assumptions of Lemma 1.1 hold. Let

$$
\gamma(y)=\theta(y)=\min _{t \in[0, T]} y(t), \quad \alpha(y)=\|y\|, \quad y \in P .
$$

Obviously, $\gamma, \alpha$ are increasing nonnegative continuous functionals on $\mathrm{P}$, and $\theta$ is nonnegative continuous functionals on $\mathrm{P}$ with $\theta(0)=0$. Moreover, we have

$$
\gamma(y)=\theta(y) \leqslant \alpha(y), \quad\|y\| \leqslant \frac{1}{\delta} \gamma(y), \quad y \in P,
$$

and

$$
\theta(\lambda y)=\lambda \theta(y), \quad \lambda \in[0,1], \quad y \in \partial P\left(\theta, b_{1}\right)
$$

Define an operator on $\mathrm{C}_{\mathrm{T}}(\mathbb{R})$ as follows:

$$
(A y)(t)=\int_{t}^{t+T} G(t, s)[b(s) f(s, y(s-\tau(s)))+c(s) g(s, y(\gamma(s)))] d s, \quad t \in \mathbb{R}, \quad y \in C_{T}(\mathbb{R}) .
$$

Let us show that $A$ is a completely continuous operator from $\overline{P\left(\gamma, c_{1}\right)}$ to P. Firstly, for every y $\in C_{T}(\mathbb{R})$, noting that

$$
\mathrm{G}(\mathrm{t}+\mathrm{T}, \mathrm{s}+\mathrm{T})=\mathrm{G}(\mathrm{t}, \mathrm{s}), \quad \mathrm{t}, \mathrm{s} \in \mathbb{R},
$$

and $s \mapsto b(s) f(s, y(s-\tau(s)))+c(s) g(s, y(\gamma(s)))$ is T-periodic, we conclude that Ay is T-periodic. Secondly, by using the dominated convergence theorem, it is not difficult to show that for every $y \in C_{T}(\mathbb{R}),(A y)(t)$ is continuous on $\mathbb{R}$. Thus, $A\left(C_{T}(\mathbb{R})\right) \subset C_{T}(\mathbb{R})$. Thirdly, for every $y \in C_{T}(\mathbb{R})$, there holds

$$
\begin{aligned}
A y(t) & =\int_{t}^{t+T} G(t, s)[b(s) f(s, y(s-\tau(s)))+c(s) g(s, y(\gamma(s)))] d s \\
& \geqslant M_{1} \int_{t}^{t+T}[b(s) f(s, y(s-\tau(s)))+c(s) g(s, y(\gamma(s)))] d s \\
& \geqslant \frac{M_{1}}{M_{2}}\|A y\|=\delta\|A y\|,
\end{aligned}
$$

where $\|A y\| \leqslant M_{2} \int_{t}^{t+T}[b(s) f(s, y(s-\tau(s)))+c(s) g(s, y(\gamma(s)))] d s$. This means that $A$ maps $C_{T}(\mathbb{R})$ to $P$.

Fourthly, noting that for every $x, y \in C_{T}(\mathbb{R})$,

$$
\|A x-A y\|=\sup _{t \in[0, T]}\left|\int_{t}^{t+T} G(t, s) \mathcal{F}(s) d s\right|,
$$


where

$$
\mathcal{F}(s)=b(s)[f(s, x(s-\tau(s)))-f(s, y(s-\tau(s)))]+c(s)[g(s, x(\gamma(s)))-g(s, y(\gamma(s)))],
$$

since $f, g$ are uniformly continuous on compact subsets, we conclude that $A: C_{T}(\mathbb{R}) \rightarrow P$ is a continuous operator. Finally, let us show that $A$ maps bounded sets into relatively-compact sets. Fix $k>0$. It is easy to see that

$$
\left\{(A y)(t): y \in C_{T}(\mathbb{R}),\|y\| \leqslant k, t \in \mathbb{R}\right\},
$$

is uniformly bounded. Moreover, following from the sufficient part of the proof for Theorem 2.2, for every $y \in C_{T}(\mathbb{R})$,

$$
(A y)^{\prime}(t)=-a(t) A y(t)+b(t) f(t, y(t-\tau(t)))+c(t) g(t, y(\gamma(t))), \quad t \in \bigcup_{i \in \mathbb{Z}}\left(t_{i}, t_{i+1}\right),
$$

which means that

$$
\left\{(A y)^{\prime}(t): y \in C_{T}(\mathbb{R}),\|y\| \leqslant \kappa, t \in \bigcup_{i \in \mathbb{Z}}\left(t_{i}, t_{i+1}\right)\right\},
$$

is also uniformly bounded. Then, by the classical Lagrange's Mean Value Theorem, one can conclude that

$$
\left\{(A y)(t): y \in C_{T}(\mathbb{R}),\|y\| \leqslant k\right\},
$$

is equi-continuous on $\mathbb{R}$. This means that $\left\{A y: y \in C_{T}(\mathbb{R}),\|y\| \leqslant k\right\}$ is relatively-compact. Thus, $A: C_{T}(\mathbb{R}) \rightarrow P$ is completely continuous.

It remains to check (i)-(iii) in Lemma 1.1 hold. Fix an arbitrary $y \in \partial P\left(\gamma, c_{1}\right)$. Then,

$$
y(t) \geqslant \min _{t \in[0, T]} y(t)=\gamma(y)=c_{1}, \quad t \in \mathbb{R},
$$

and

$$
\|y\| \leqslant \frac{1}{\delta} y(t), \quad t \in \mathbb{R},
$$

which yields that $\|y\| \leqslant \frac{c_{1}}{\delta}$ and thus $y(t) \leqslant \frac{c_{1}}{\delta}$ for all $t \in \mathbb{R}$. This means that

$$
c_{1} \leqslant y(t-\tau(t)), y(\gamma(t)) \leqslant \frac{1}{\delta} c_{1}, \quad t \in \mathbb{R},
$$

which together with (H1) implies that

$$
\begin{aligned}
\gamma(A y) & =\min _{t \in[0, T]} \int_{t}^{t+T} G(t, s)[(b(s) f(s, y(s-\tau(s)))+c(s) g(s, y(\gamma(s))))] d s \\
& \geqslant M_{1} \int_{0}^{T}[(b(s) f(s, y(s-\tau(s)))+c(s) g(s, y(\gamma(s)))] d s \\
& \geqslant M_{1} \int_{0}^{T}\left[b(s) \inf _{c_{1} \leqslant u \leqslant \frac{c_{1}}{\delta}} f(s, u)+c(s) \inf _{c_{1} \leqslant u \leqslant \frac{c_{1}}{\delta}} g(s, u)\right] d s \\
& >c_{1} .
\end{aligned}
$$

This shows that (i) of Lemma 1.1 holds. For every $y \in \partial P\left(\theta, b_{1}\right)$, similar to the above proof, we have

$$
b_{1} \leqslant y(t-\tau(t)), y(\gamma(t)) \leqslant \frac{1}{\delta} b_{1}, \quad t \in \mathbb{R} .
$$

Combining this with (H2), we get

$$
\theta(A y)=\min _{t \in[0, T]} \int_{t}^{t+T} G(t, s)[b(s) f(s, y(s-\tau(s)))+c(s) g(s, y(\gamma(s)))] d s
$$




$$
\begin{aligned}
& \leqslant M_{2} \int_{0}^{T}[b(s) f(s, y(s-\tau(s)))+c(s) g(s, y(\gamma(s)))] d s
\end{aligned}
$$

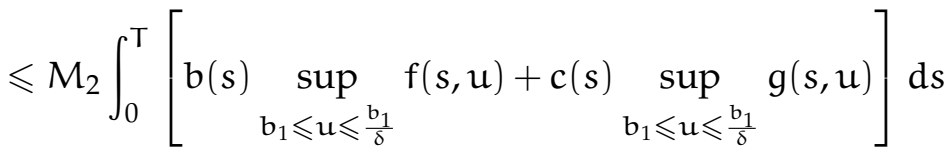

$$
\begin{aligned}
& <\mathrm{b}_{1} \text {, }
\end{aligned}
$$

i.e., (ii) of Lemma 1.1 holds. Obviously, $P\left(\alpha, a_{1}\right) \neq \emptyset$. For every $y \in \partial P\left(\alpha, a_{1}\right)$, we have

$$
\delta a_{1} \leqslant y(t-\tau(t)), y(\gamma(t)) \leqslant a_{1}, \quad t \in \mathbb{R},
$$

which together with $(\mathrm{H} 3)$ gives that

$$
\begin{aligned}
\alpha(A y) & =\sup _{t \in[0, T]} \int_{t}^{t+T} G(t, s)[b(s) f(s, y(s-\tau(s)))+c(s) g(s, y(\gamma(s)))] d s \\
& \geqslant M_{1} \int_{0}^{T}\left[b(s) \inf _{0 \leqslant u \leqslant a_{1}} f(s, u)+c(s) \inf _{0 \leqslant u \leqslant a_{1}} g(s, u)\right] d s \\
& >a_{1} .
\end{aligned}
$$

This proves (iii) of Lemma 1.1. Now, by applying Lemma 1.1, the following integral equation

$$
y(t)=\int_{t}^{t+T} G(t, s)[b(s) f(s, y(s-\tau(s))+c(s) g(s, y(\gamma(s)))] d s, \quad t \in \mathbb{R},
$$

has at least two nonnegative T-periodic solutions $x, y$, which are just two nonnegative T-periodic solutions to equation (1.1) by Lemma 2.2.

\subsection{Existence of three nonnegative solutions}

In this section, we will apply Lemma 1.2 to equation (1.1). Also, we list some assumptions.

(H4) There exists a constant $c_{1}>0$ such that

$$
\int_{0}^{T}\left[b(s) \sup _{0 \leqslant u \leqslant c_{1}} f(s, u)+c(s) \sup _{0 \leqslant u \leqslant c_{1}} g(s, u)\right] d s<\frac{c_{1}}{M_{2}} .
$$

(H5) There exist $c_{2}>c_{1}>0$ and $c_{3}>\frac{c_{2}}{\delta}$ such that

$$
\int_{0}^{T}\left[b(s) \inf _{c_{2} \leqslant u \leqslant c_{3}} f(s, u)+c(s) \inf _{c_{2} \leqslant u \leqslant c_{3}} g(s, u)\right] d s>\frac{c_{2}}{M_{1}} .
$$

(H6) There exists a constant $c_{4} \geqslant c_{3}$ such that

$$
\int_{0}^{T}\left[b(s) \sup _{0 \leqslant u \leqslant c_{4}} f(s, u)+c(s) \sup _{0 \leqslant u \leqslant c_{4}} g(s, u)\right] d s \leqslant \frac{c_{4}}{M_{2}} .
$$

Theorem 2.4. Assume that (H4)-(H6) hold. Then (1.1) has at least three nonnegative T-periodic solutions.

Proof. Let the cone P and the operator A be the same as in Theorem 2.3. Set

$$
\psi(y)=\min _{t \in[0, T]} y(t), \quad y \in P .
$$


Obviously, $\psi$ is a concave nonnegative continuous functional on $\mathrm{P}$ and $\psi(\mathrm{y}) \leqslant\|y\|$ for all $y \in P$. By the proof of Theorem 2.3, we know that $A: C_{T}(\mathbb{R}) \rightarrow P$ is completely continuous. Moreover, by (H6), for every $y \in \overline{\mathrm{P}_{\mathrm{c}_{4}}}$, we have

$$
\begin{aligned}
\|A y\| & =\sup _{t \in[0, T]} \int_{t}^{t+T} G(t, s)[b(s) f(s, y(s-\tau(s)))+c(s) g(s, y(\gamma(s)))] d s \\
& \leqslant M_{2} \int_{0}^{T}\left[b(s) \sup _{0 \leqslant u \leqslant c_{4}} f(s, u)+c(s) \sup _{0 \leqslant u \leqslant c_{4}} g(s, u)\right] d s \\
& \leqslant c_{4} .
\end{aligned}
$$

Next, let us verify (i)-(iii) of Lemma 1.2. Firstly, it is easy to see that

$$
\left\{u \in P\left(\psi, c_{2}, c_{3}\right): \psi(u)>c_{2}\right\}=\left\{u \in P: \psi(u)>c_{2},\|u\| \leqslant c_{3}\right\} \neq \varnothing .
$$

Moreover, for every $y \in P\left(\psi, c_{2}, c_{3}\right)$, there holds

$$
c_{2} \leqslant y(t-\tau(t)), y(\gamma(t)) \leqslant c_{3}, \quad t \in \mathbb{R} .
$$

Combining this with (H5), we get

$$
\begin{aligned}
\psi(A y) & =\min _{t \in[0, T]} \int_{t}^{t+T} G(t, s)[b(s) f(s, y(s-\tau(s)))+c(s) g(s,(\gamma(s)))] d s \\
& \geqslant M_{1} \int_{0}^{T}\left[b(s) \inf _{c_{2} \leqslant u \leqslant c_{3}} f(s, u)+c(s) \inf _{c_{2} \leqslant u \leqslant c_{3}} g(s, u)\right] d s \\
& >c_{2} .
\end{aligned}
$$

Secondly, it follows from $(\mathrm{H} 4)$ that for every $y \in \overline{\mathrm{P}_{\mathrm{c}_{1}}}$,

$$
\begin{aligned}
\|A y\| & =\sup _{t \in[0, T]} \int_{t}^{t+T} G(t, s)[b(s) f(s, y(s-\tau(s)))+c(s) g(s, y(\gamma(s)))] d s \\
& \leqslant M_{2} \int_{0}^{T}\left[b(s) \sup _{0 \leqslant u \leqslant c_{1}} f(s, u)+c(s) \sup _{0 \leqslant u \leqslant c_{1}} g(s, u)\right] d s \\
& <c_{1} .
\end{aligned}
$$

Finally, $y \in P$ with $\|A y\|>c_{3}$ implies that

$$
\psi(A y)=\min _{t \in[0, T]}(A y)(t) \geqslant \delta\|A y\| \geqslant \delta c_{3}>c_{2}
$$

This means that (iii) of Lemma 1.2 holds.

Now, by Lemma 1.2, we know that $A$ has at least three fixed points, and thus (1.1) has at least three nonnegative T-periodic solutions.

\subsection{An example}

Stimulated by [12], in this section, we apply one of our main results to the following Nicholson's blowflies type model:

$$
x^{\prime}(t)=-a(t) x(t)+p(t) x^{m}(\gamma(t)) e^{-q(t) x^{n}(\gamma(t))},
$$

where $m>1, n>0, a, p, q$ are nonnegative continuous T-periodic functions and let $\gamma$ be the same as in Section 1.

Recall that Nicholson's blowflies model has an interesting background (cf. [8]). Recently, several authors have made contributions on the existence of multiple periodic solutions for Nicholson's blowflies type model (cf. $[7,12,13])$. Equation $(2.5)$ can be seen as a generalized Nicholson's blowflies type model, which allows the delay term to be a discontinuous function. 
Theorem 2.5. Let $\mathrm{q}(\mathrm{t})>0$ for all $\mathrm{t} \in \mathbb{R}$, and

$$
\int_{0}^{T} p(t) d t>\frac{e\|q\|^{\frac{m-1}{n}}}{\delta^{m-1}}
$$

where $\delta$ is the same as in Theorem 2.3. Then (2.5) has at least two positive T-periodic solutions.

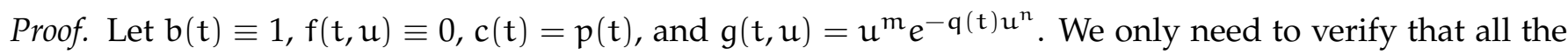
assumptions of Theorem 2.4 are satisfied.

Firstly, we can choose sufficiently small $c_{1}>0$ such that

$$
\mathrm{c}_{1}^{\mathrm{m}} \int_{0}^{\mathrm{T}} \mathrm{p}(\mathrm{s}) \mathrm{d} \mathrm{s}<\frac{\mathrm{c}_{1}}{\mathrm{M}_{2}}
$$

which yields that

$$
\int_{0}^{T} p(s) \sup _{0 \leqslant u \leqslant c_{1}} u^{m} e^{-q(s) u^{n}} d s \leqslant c_{1}^{m} \int_{0}^{T} p(s) d s<\frac{c_{1}}{M_{2}}
$$

i.e., (H4) holds.

Secondly, since

$$
\int_{0}^{T} p(t) d t>\frac{e\|q\|^{\frac{m-1}{n}}}{\delta^{m-1}}
$$

we can choose $\lambda \in(0,1)$ such that

$$
\int_{0}^{T} p(t) d t>\frac{e\|q\|^{\frac{m-1}{n}}}{\lambda^{m-1} \delta^{m-1}} .
$$

Letting $c_{2}=\frac{\lambda \delta}{\|q\|^{\frac{1}{n}}}$ and $c_{3}=\frac{1}{\|q\|^{\frac{1}{n}}}$, we have

$$
\int_{0}^{T} p(s) \inf _{c_{2} \leqslant u \leqslant c_{3}} u^{m} e^{-q(s) u^{n}} d s \geqslant \int_{0}^{T} p(s) d s \cdot c_{2}^{m} \cdot e^{-\|q\| c_{3}^{n}}>c_{2},
$$

which means that (H5) holds.

Finally, letting $\varepsilon=\min _{t \in[0, T]} q(t)$, since

$$
\lim _{u \rightarrow+\infty} \frac{u^{m} e^{-\varepsilon u^{n}}}{u}=0<\frac{1}{2 M_{2} \int_{0}^{T} p(s) d s},
$$

there exists $K>0$ such that for all $u \geqslant K$, there holds

$$
\int_{0}^{T} p(s) d s \cdot u^{m} e^{-\varepsilon u^{n}}<\frac{u}{2 M_{2}} .
$$

Let $c_{4}=\max \left\{M_{2} K^{m} \int_{0}^{T} p(s) d s+1, c_{3}\right\}$. Then, (H6) holds, i.e.,

$$
\begin{aligned}
\int_{0}^{T} p(s) \sup _{0 \leqslant u \leqslant c_{4}} u^{m} e^{-q(s) u^{n}} d s & \leqslant \max \left\{\int_{0}^{T} p(s) \sup _{0 \leqslant u \leqslant K} u^{m} e^{-q(s) u^{n}} d s, \int_{0}^{T} p(s) \sup _{K \leqslant u \leqslant c_{4}} u^{m} e^{-q(s) u^{n}} d s\right\} \\
& \leqslant \max \left\{\int_{0}^{T} p(s) d s \cdot K^{m}, \sup _{K \leqslant u \leqslant c_{4}} \int_{0}^{T} p(s) d s \cdot u^{m} e^{-\varepsilon u^{n}}\right\} \\
& <\frac{c_{4}}{M_{2}} .
\end{aligned}
$$

Thus, by Theorem 2.4, equation (2.5) has at least two positive T-periodic solutions since 0 is obviously a solution to equation (2.5). 
Remark 2.6. One of the key assumptions in [12] is (translated in our notations):

$$
\int_{0}^{T} p(t) d t>\left(\frac{1}{\delta}-1\right) \cdot \frac{e\|q\|^{\frac{m-1}{n}}}{\delta^{m-1}} .
$$

Here, (2.6) improves it to some extent (in the case of $\delta=e^{-\int_{0}^{T} a(t) d t}$ is sufficiently small).

\section{Acknowledgment}

The work was partially supported by NSFC (11461034), the Program for Cultivating Young Scientist of Jiangxi Province (20133BCB23009), the NSF of Jiangxi Province (20143ACB21001), and the Foundation of Jiangxi Provincial Education Department (GJJ150342).

\section{References}

[1] M. U. Akhmet, Stability of differential equations with piecewise constant arguments of generalized type, Nonlinear Anal., 68 (2008), 794-803. 1

[2] M. U. Akhmet, Quasilinear retarded differential equations with functional dependence on piecewise constant argument, Commun. Pure Appl. Anal., 13 (2014), 929-947. 1

[3] R. I. Avery, J. Henderson, Two positive fixed points of nonlinear operators on ordered Banach spaces, Comm. Appl. Nonlinear Anal., 8 (2001), 27-36. 1, 1.1

[4] S. Castillo, M. Pinto, Existence and stability of almost periodic solutions to differential equations with piecewise constant arguments, Electron. J. Differential Equations, 2015 (2015), 15 pages. 1

[5] K.-S. Chiu, J.-C. Jeng, Stability of oscillatory solutions of differential equations with general piecewise constant arguments of mixed type, Math. Nachr., 288 (2015), 1085-1097.

[6] K.-S. Chiu, M. Pinto, J.-C. Jeng, Existence and global convergence of periodic solutions in recurrent neural network models with a general piecewise alternately advanced and retarded argument, Acta Appl. Math., 133 (2014), 133-152. 1

[7] H.-S. Ding, J. G. Dix, Multiple periodic solutions for discrete Nicholson's blowflies type system, Abstr. Appl. Anal., 2014 (2014), 6 pages. 2.4

[8] W. S. C. Gurney, S. P. Blythe, R. M. Nisbet, Nicholson's blowflies revisited, Nature, 287 (1980), 17-21. 2.4

[9] M. N. Islam, Y. N. Raffoul, Periodic solutions of neutral nonlinear system of differential equations with functional delay, J. Math. Anal. Appl., 331 (2007), 1175-1186. 2.1

[10] W. G. Jian, Y. Y. Chen, Pseudo almost periodic type solutions to a class of nonlinear difference equations, J. Jiangxi Normal Univ. (Natural Science Edition), (to appear). 1

[11] R. W. Leggett, L. R. Williams, Multiple positive fixed points of nonlinear operators on ordered Banach spaces, Indiana Univ. Math. J., 28 (1979), 673-688. 1, 1.2

[12] S. Padhi, S. Pati, Positive periodic solutions for a nonlinear functional differential equation, Mem. Differential Equations Math. Phys., 51 (2010), 109-118. 2.4, 2.4, 2.6

[13] S. Padhi, S. Srivastava, J. G. Dix, Existence of three nonnegative periodic solutions for functional differential equations and applications to hematopoiesis, PanAmer. Math. J., 19 (2009), 27-36. 2.4

[14] M. Pinto, Asymptotic equivalence of nonlinear and quasi linear differential equations with piecewise constant arguments, Math. Comput. Modelling, 49 (2009), 1750-1758. 1

[15] M. Pinto, Cauchy and Green matrices type and stability in alternately advanced and delayed differential systems, J. Difference Equ. Appl., 17 (2011), 235-254.

[16] T. Veloz, M. Pinto, Existence, computability and stability for solutions of the diffusion equation with general piecewise constant argument, J. Math. Anal. Appl., 426 (2015), 330-339. 1

[17] J. Wiener, Oscillations and multiple periodic solutions in systems of differential equations with piecewise continuous delay, Appl. Anal., 74 (2000), 289-299. 1 